\title{
Effect of Noise on Frequency and Time Domain Methods of T Wave Alternans
}

\author{
JQ Xue, GI Rowlandson \\ GE Healthcare, Milwaukee, Wisconsin, USA
}

\begin{abstract}
The purpose of this study is to objectively evaluate the detection accuracy of $T$ Wave Alternans (TWA) methods with a simulation platform and different simulated noise sources including muscle noise, baseline wander, beat alignment error and possible physiological TWA shift modulated by $R R$ interval variations. We designed several new methods for time-domain TWA evaluation including a new clustering analysis and a TWA timeseries analysis.

We demonstrated that the alignment error which can be caused by beat detection error and/or by beat-to-beat physiological variations can be one of the most severe noise sources for microvolt TWA. We also demonstrated that a new preprocessing method based on principal component analysis (PCA) can compensate the TWA alignment error and improve detection accuracy by more than 50\% for both frequency and time domain methods.
\end{abstract}

\section{Introduction}

Microvolt $\mathrm{T}$ wave alternans (TWA) has been found useful to predict life-threatening arrhythmia events and therefore could also be useful in identifying candidates for implantable cardioverter defibrillators. Current $\mathrm{T}$ Wave Alternans (TWA) detection algorithms work in two different domains: time and frequency. Both methods try to detect microvolt TWA appearing on surface ECGs during stress testing. The time domain method has also been used on Holter data. Due to very small TWA amplitudes and possible severe background noise including muscle noise, baseline wander, etc, it is very difficult to verify TWA detection from the displayed or the printed ECG waveforms. Special signal-to-noise ratio (SNR) improvement and signal processing are needed to enhance the SNR and to facilitate visualization of the TWA.

The frequency domain method uses spectral analysis to enhance the alternans pattern at the half of the beat frequency [1]. On the other hand, the time domain method enhances the SNR by using incremental median odd and even beats [2].

It is important to have a direct and objective method to evaluate the microvolt TWA detection algorithms. With such a simulation platform, we should be able to evaluate TWA detection under different signal and noise circumstances. For example, different $\mathrm{T}$ wave shape and TWA amplitude, different types of noise.

The paired-t test is a tool for evaluation of the hypothesis that two group of measurements are significantly different statistically [3]. Here we use this method to evaluate the $\mathrm{T}$ waves of alternate beats for a significant difference. The parameters to be examined can be any useful features from the ST-T wave, e.g. amplitude, area, slope, and other morphology related parameters.

Principal component analysis (PCA) is an effective method to enhance the signal-to-noise ratio in many signal processing tasks including ECG signal detection [4]. The basic idea of using PCA for TWA detection is to find if noise effect can be decrease by using dominant PCA vector formed from ST-T segment.

\section{Methods}

\subsection{Simulation signal and model}

A simulation platform is designed for evaluation of TWA detection under different signal and noise circumstances. Some of the simulation parameters are:

- T wave model: The $\mathrm{T}$ wave is generated as: $A \cos ^{\alpha}\left(\frac{2 \pi t}{T}\right)$, where A controls the $\mathrm{T}$ wave amplitude, T controls the width, and power factor $\alpha$ controls the general shape of the $\mathrm{T}$ wave.

- TWA: TWA is added to alternate beats by changing the amplitude coefficient A, or the power factor $\alpha$.

- Muscle noise: Muscle noise is generated by color filtered (bandpass) random noise.

- Alignment error: Alignment error can come from either physiologic change of the $\mathrm{T}$ wave on a beat by beat basis, or from fiducial point jitter from the beat detection (QRS detection) algorithms. Alignment error is simulated with a random shift of each beat to its original aligned position.

- Baseline wander: Baseline wander is simulated with a low frequency sinusoidal wave, which is added to the simulated $\mathrm{T}$ waves. 
Also simulated are sampling frequency, resolution of digitization, number of cardiac cycle used for TWA ( 32 , $64,128)$.

A simulated ECG waveform with $50 \mathrm{uV}$ of $\mathrm{T}$ wave amplitude alternans (peak-to-peak) added is shown in Figure 1.

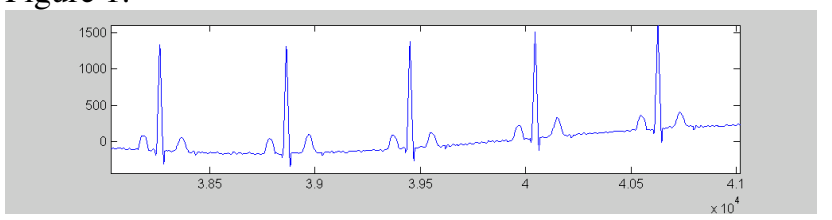

Figure 1. A simulated ECG waveform with $\mathrm{T}$ wave alternans and different type of noise added.

\subsection{Verification methods}

The verification methods are divided into time and frequency domains and the focus is on the time domain methods. The study presented here addresses both estimation of TWA amplitude and the confidence of the detection. The new evaluation methods are designed for providing the confidence level check.

\subsubsection{Time domain:}

\section{Incremental median of odd and even beats}

The basis for measuring TWA in the time domain is the formation of a median PQRST complex for odd and even beats [2]. The median beat complex is formed in a way to minimize the effects of random noise and allow comparison of the every-other-beat $\mathrm{T}$ wave fluctuation by comparing ST measurement in the ST-T segment. The key process in this method is signal conditioning to reduce noise effects, rejection of ectopic beats, and formation of a median PQRST complex for the odd and even beat. The odd and the even median beats are updated by either a fixed increment, or a fraction of the difference between the median template and the incoming beat, whichever is less. The default update fraction is $1 / 32$, but other factors are selectable, namely $1 / 8,1 / 16$ or $1 / 64$.

Figure 2 shows an example of odd and even beat median formation from individual beats of $\mathrm{T}$ wave. After median formation, the alternans can be clearly observed and measured.

\section{Clustering analysis on $T$ wave feature map}

A clustering analysis is designed on a two dimensional ST-T feature map, i.e. Beat +1 vs. Beat (Lorenz plot). The separation of the two groups (odd and even beats) can be measured by using such statistical methods as the paired $t$ test. This separation can also be viewed graphically as shown in Figure 3, where the difference of the odd and even beat features can be observed through obvious separation of two clusters, or it can be also stated that the intra-cluster variance is statistically smaller than intercluster variance. At the same time, the alternans amplitude can be estimated by measuring the distance from the centers of two clusters.

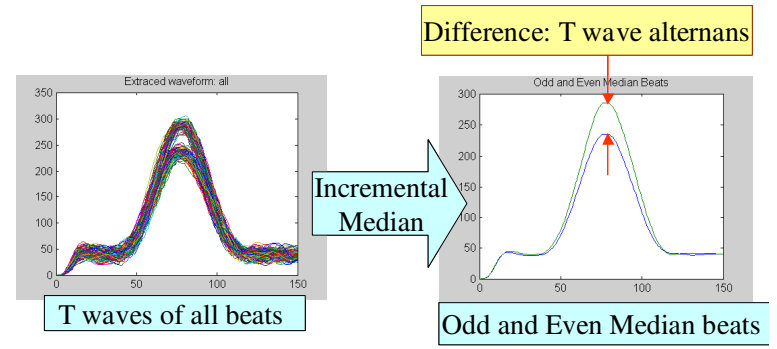

Figure 2. Time domain representation of TWA: Median even and odd beats.

This plot can be further extended to the Beat $+\mathrm{K}$ vs. Beat plot, where $\mathrm{K}$ is the beat interval to be examined. For example, for an $\mathrm{ABAB}$ alternans pattern, we would assume that the Beat+2 vs. Beat plot will not show separation of clusters and Beat +3 vs. Beat plot will. This extension can also be used to analyze other alternans pattern other than an $\mathrm{ABAB}$ pattern.

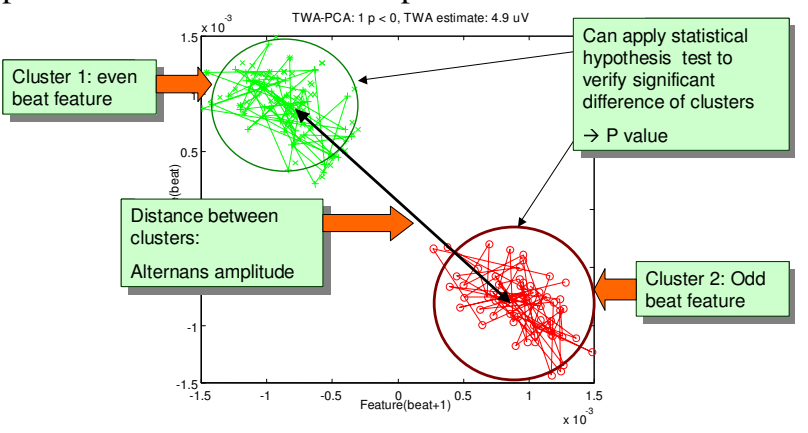

Figure 3. Clustering and paired $t$ statistical tests for TWA detection.

\section{Trend of alternans beat and non-alternans beat curves}

A time series trend of TWA estimation is plotted in Figure 4. In the Figure, in addition to the trending of TWA estimation based on both even and odd beats, we also plot TWA estimation of even beats only or odd beat only. The assumption is that if TWA is presented as $\mathrm{ABAB}$ pattern, the curve including both even and odd beats should have a good separation from the one including only even(or odd) beats. If the two curves do not show significant separation, TWA detection is not significant. A histogram of two trending curves can be 
used to find significance of the TWA detection.

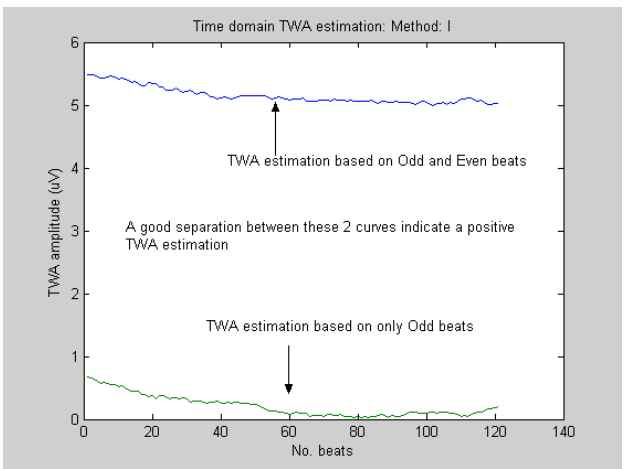

Figure 4. A trend plot of time domain TWA estimation.

\subsubsection{Frequency domain}

The evaluation of the frequency domain method is based on the scoring method described in [1]. A score, K, is defined as the value of the spectrum at the half beat (0.5 cycles/beat) minus the baseline spectrum and normalized by the standard deviation of the baseline spectrum, where the baseline spectrum is the mean spectrum value from 0.44 to 0.49 cycles/beat.

$\mathrm{K}=(\operatorname{Spec}(0.5)-\operatorname{Mean}(\operatorname{Spec}(0.44: 0.48)) / \operatorname{STD}($ $\operatorname{Spec}(0.44: 0.49))$

If $\mathrm{K}$ is larger than 3 , define TWA as positive, otherwise negative.

\subsection{Noise effects}

In this presentation, we will focus on muscle noise and alignment error, although there are other type of noise, such as baseline wandering and footfall noise as in stress test cases. In the example shown in Figure 5, a 10 $\mathrm{uV}$ peak-to-peak (p-p) TWA is added to alternate T waves. The noise level is increased from 20 to $200 \mathrm{uV}$ (rms). It can be seen that detection of TWA gets worse for both the time and frequency domain methods, although the time domain clustering analysis still shows significant difference of odd and even beat clusters when the noise reaches $200 \mathrm{uV}$.

In the alignment noise case, we added an amount of random variation from the QRS detection trigger point to the $\mathrm{T}$ wave onset point to simulate the alignment error caused by either variation of QRS detection and /or physiological variation of phase of alternans from beat to beat. Both of these situations can happen in real measurements. In the example shown in Figure 6, when alignment error is increased to $20 \mathrm{msec}$, the confidence level dropped below the thresholds for both time and frequency domain methods, although the spectrum method suffered more with this type of error as its $\mathrm{K}$ value decayed more dramatically.

\section{Muscle noise}

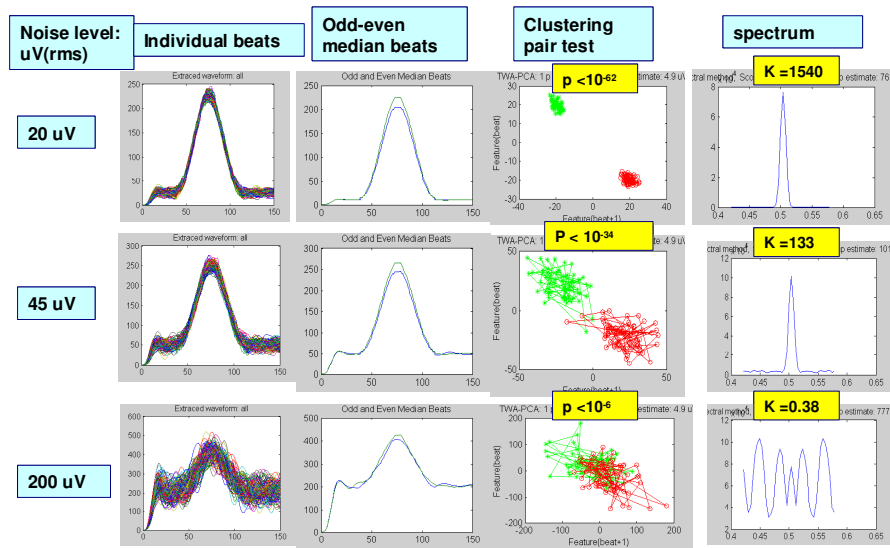

Figure 5. The effects of muscle noise on detection of TWA.

Alignment noise

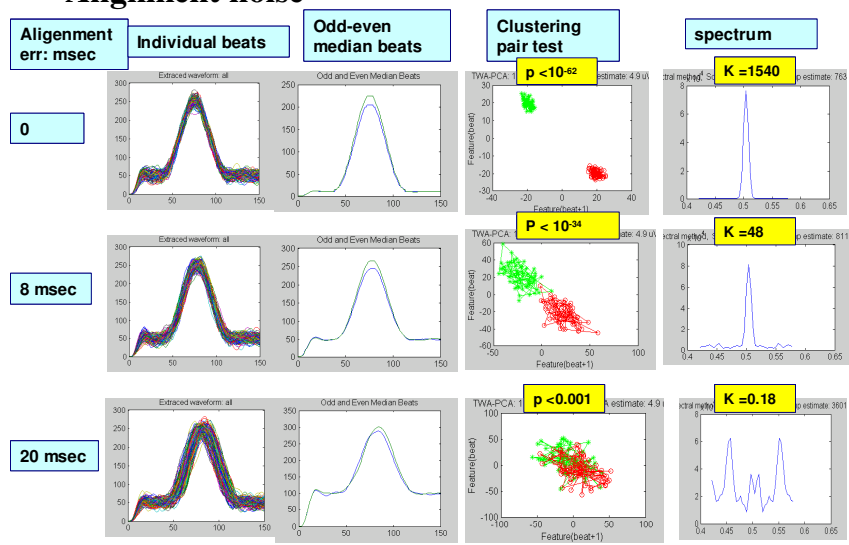

Figure 6. Effects of alignment error on TWA detection.

\subsection{PCA based preprocessing to improve robustness of TWA estimation}

In this preprocessing method, a matrix $\mathbf{A}$ is formed by sampling the entire $\mathrm{T}$ wave (or any defined segment in the ST-T wave). In matrix $\mathbf{A}$, each row corresponds to one beat, and each column corresponds to a selected feature of the ST-T wave. The amplitude is the simplest feature. The matrix $\mathrm{A}$ is then decomposed into 3 matrices, $\mathrm{U}, \mathrm{S}$, and $\mathrm{V}$ by the singular-valuedecomposition (SVD) [5].

$$
A=U S V^{T}
$$

where $\mathrm{U}$ and $\mathrm{V}$ are orthonormal matrices as $\left[u_{1}, u_{1}, \ldots . u_{k}\right]$ and $\left[v_{1}, v_{1}, \ldots . v_{k}\right], \quad \mathrm{S}$ is a diagonal matrix called the singular matrix and can be denoted $\left[\mathrm{s}_{1}\right.$, $\left.s_{2}, \quad \ldots \quad s_{n}\right]_{\text {diag }}$, whose elements have monotonically decreasing values, i.e. $s_{1}>s_{2}>\ldots>s_{n}$. This is shown graphically in Figure 7 . The first principal vector $u_{1}$ contains the most dominant variance from beat-to-beat 
and is therefore used for TWA analysis.

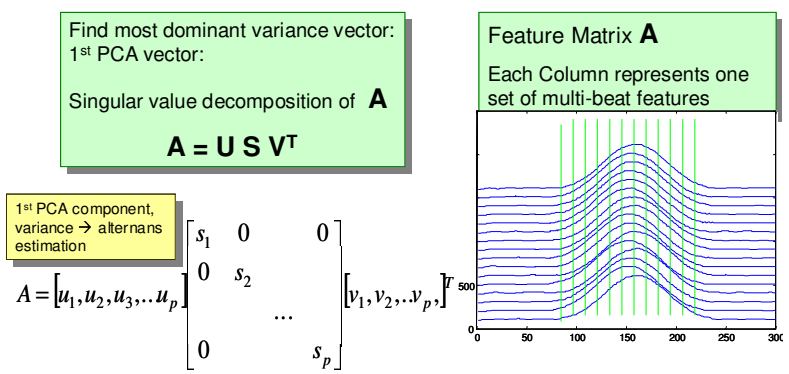

Figure 7. The formation of matrix from selected ST-T segment and singular-value-decomposition of the matrix.

\section{Results}

In Figure 8, we demonstrate in two cases of noisy signal where the time and frequency domain methods both show poor detection of $10 \mathrm{uV}$ TWA added to the simulated ECG waveform. After applying PCA preprocessing to the signal, the paired test show a much more significant separation of odd and even beat clusters, and the spectrum peak around half the beat frequency is also much sharper (where $\mathrm{K}$ value $>3$ means positive TWA detection).

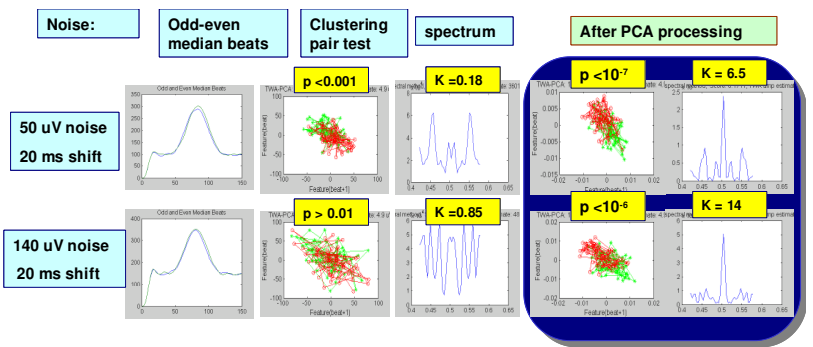

Figure 8. Improving TWA detection by applying PCA preprocessing to noisy ECG data.

Using these principles, a Monte-Carlo simulation analysis was conducted. In this test, the TWA amplitudes (rms)ranged from 0 (no TWA) to $50 \mathrm{uV}$. The muscle noise amplitude (rms) ranged from 20 to $250 \mathrm{uV}$, and the baseline wander ranged from 100 to $1000 \mathrm{uV}$, and finally the alignment error ranged from 0 to $16 \mathrm{msec}$. A total of 310 data sets were created. The time domain method of TWA was used, and the confidence level was checked with the paired T test with 32 beats in each cluster. The threshold for positive TWA detection (significant difference between two clusters) was $\mathrm{p}<0.0001$.

The results of the Monte Carlo analysis are summarized in Figure 9, which shows $100 \%$ specificity for both with and without PCA preprocessing. With PCA preprocessing, the sensitivity doubled at $2 \mathrm{uV}$ TWA detection to $60 \%$ and increased to $100 \%$ for $10 \mathrm{uV} \mathrm{TWA.}$

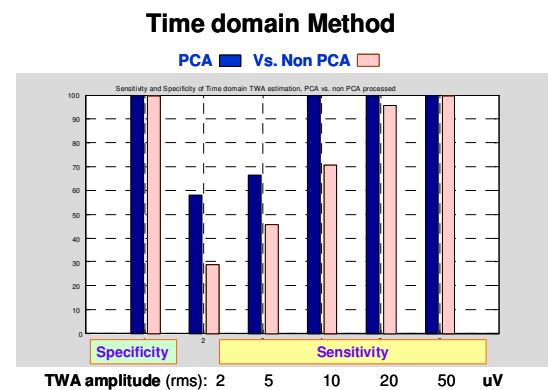

Figure 9. The results show $100 \%$ specificity for both with and without PCA preprocessing. With PCA preprocessing, sensitivity is doubled at $2 \mathrm{uV}$ TWA detection to $60 \%$ and increases to $100 \%$ for $10 \mathrm{uV} \mathrm{TWA}$.

\section{Discussion and conclusions}

The results of this simulation test demonstrate that the confidence level tests based on feature map clustering provide an objective method for judging positive or negative TWA detection. We also proved through simulation that the PCA preprocessing can improve the detection of TWA for both time and frequency domain methods. In the example we can see that the sensitivity is improved in each level of TWA amplitude, especially at less than the $10 \mathrm{uV}$ level, which is most difficult to detect in stress and ambulatory cases, where ECG is relatively noisy. We also noticed that the new method maintained same high specificity while improving the sensitivity.

\section{References}

[1] Rosenbaum DS, .Jackson LE, . Smith JM, Garan H , Ruskin J, and Cohen RJ . Electrical alternans and vulnerability to ventricular arrhythmias. The New England J. Medicine, 330(4):235-241, January 271994.

[2] Nearing BD and Verrier RL. Modified moving average analysis of T-wave alternans to predict ventricular fibrillation with high accuracy. J. Appl Physiol. Vol 92, 541-549, 2002

[3] Rosner B, Fundamentals of Biostatistics, $3^{\text {rd }}$ ed. PWS-Kent publishing company, 1990.

[4] Xue QZ, Reddy BRS, "Algorithms for computerized QT analysis", J. of Electrocardiology Vol 30, Sup. 181-186, 1997.

[5] Golub GH, van Loan CF, Matrix Computation, 2nd ed. Baltimore: Johns Hopkins University Press, 1989.

Joel Xue

W126 N7449 Flint Road, Menomonee Falls, WI 53051

joel.xue@med.ge.com 